\title{
Médiévales
}

Langues, Textes, Histoire

65 | automne 2013

Le couple dans le monde franc

\section{Couples chastes à la fin de l'Antiquité et au haut Moyen Âge}

Chaste Couples in Late Antiquity and in the Early Middle Ages

Sylvie Joye

\section{(2) OpenEdition}

1 Journals

Édition électronique

URL : https://journals.openedition.org/medievales/7090

DOI : 10.4000/medievales. 7090

ISSN : 1777-5892

Éditeur

Presses universitaires de Vincennes

Édition imprimée

Date de publication : 1 décembre 2013

Pagination : 47-63

ISBN : 978-2-84292-396-9

ISSN : 0751-2708

Référence électronique

Sylvie Joye, "Couples chastes à la fin de l'Antiquité et au haut Moyen Âge », Médiévales [En ligne], 65 | automne 2013, mis en ligne le 20 janvier 2014, consulté le 22 avril 2022. URL : http://

journals.openedition.org/medievales/7090; DOI : https://doi.org/10.4000/medievales.7090

Tous droits réservés 
Médiévales 65, automne 2013,p. 47-64

Sylvie JOYE

\section{COUPLES CHASTES À LA FIN DE L'ANTIQUITÉ ET AU HAUT MOYEN ÂGE}

La sexualité apparaît comme l'un des éléments essentiels à la définition du couple, au très haut Moyen Âge, tout comme à d'autres époques ${ }^{1}$. Un rôle fondateur a même été assigné à celle-ci dans le mariage ou le concubinage altomédiéval par beaucoup d'historiens, et même par des auteurs carolingiens tel Hincmar, lorsqu'ils ont tenté de définir les unions qui contrevenaient aux nouvelles formes du mariage chrétien ${ }^{2}$. Si la part de la sexualité, du consentement ou de la religion a pu être variable dans le rapport entre les époux, et si le mariage est demeuré avant tout une affaire conclue entre deux familles, le couple chaste ${ }^{3}$ se définit néanmoins comme une entité qui permet aux individus de se construire via leur attitude et leur discours par rapport à la sexualité et à la religion.

1. Par exemple I. THÉRY, Couple, filiation et parenté aujourd'hui. Le droit face aux mutations de la famille et de la vie privée, Paris, 1998, p. 143.

2. S. JoYE, «Y a-t-il une "évolution" des mœurs? Historiographie et anthropologie de la famille et du mariage », Labyrinthe, 30 (2008), p. 115-130; J. GAUDEMET, «Indissolubilité et consommation du mariage : l'apport d'Hincmar de Reims », Revue de droit canonique, 30 (1980), p. 28-40; P. Toxé, «La copula carnalis chez les canonistes médiévaux», dans M. Rouche éd., Mariage et sexualité au Moyen Âge, Paris, 2000, p. 123-133.

3. L'expression peut souligner dans les sources latines la pureté et la perfection spirituelle des personnes, selon l'usage répandu du terme castitas dans la doctrine chrétienne, mais nous traiterons ici des couples chastes tels qu'ils ont été entendus par le père de Gaiffier dans son article séminal ( $c f$. n. 44), et nous concentrons même plus spécifiquement sur les couples réputés avoir conservé leur virginité dans le mariage d'après leurs hagiographes. Notons que certains auteurs ont aussi avancé avec raison que la définition de la virginité est entendue parfois au Moyen Âge d'un point de vue plus spirituel que biologique (F. Lifshitz, «Priestly Women, Virginal Men», dans L. Bitel, F. Lifshitz éd., Gender and Christianity in Medieval Europe, University of Pennsylvania Press, 2008, p. 87-95), mais les récits qui nous retiennent ici semblent unir fermement les deux aspects et insister sur l'aspect primordial de la préservation effective de la virginité. 
Ici, il s'agit bien entendu davantage de l'image que l'hagiographe veut donner du couple que des actes et discours véritables des couples présentés comme chastes. Même si des couples ont bien fait vie et lit communs tout en refusant la pratique effective de la sexualité à la fin de l'Antiquité et au très haut Moyen Âge, les sources hagiographiques nous délivrent une mise en scène très précise de scènes intimes qui ne peuvent être que des créations de l'hagiographe. Par ailleurs, comme toujours en ce qui concerne les saints, et en particulier leur vie de famille, les hagiographes cherchent avant tout à mettre en avant des idéaux de sainteté ${ }^{4}$. On ne peut parler que partiellement de modèles cependant, en ce qui concerne les couples chastes (en tout cas ceux qui renoncent à la sexualité dès leur formation tout en maintenant une réelle cohabitation). Ce sont ces couples qui vont retenir essentiellement notre attention ici.

\section{Les paradoxes de la valorisation de l'ascétisme et de la morale du couple à la fin de l'Antiquité}

Ramsay McMullen avait proposé de voir dans la sexualité le seul domaine où la christianisation aurait changé quelque chose ${ }^{5}$. Cette affirmation ne semble pas tout à fait exacte: disons du moins que la christianisation seule n'explique pas les modifications de la sexualité et ses rapports avec la notion de couple durant l'Antiquité tardive et le haut Moyen Âge. On se souvient de la façon dont Paul Veyne a souligné que l'essor de la «morale du couple ${ }^{6}$ est indépendant de la christianisation et antérieur à elle, et celle dont Michel Foucault, qui récuse d'ailleurs l'usage du terme même de sexualité pour cette période, a fait de même ${ }^{7}$. Une partie de leurs analyses a été remise en cause ${ }^{8}$. Ruth Mazo Karras critique de façon convaincante la restriction chronologique assignée par Foucault au terme de «sexualité » et cherche à démontrer qu'il peut être appliqué au Moyen Âge.

4. G. PhilipPART, «Le riche et encombrant héritage de Jean Bolland (1643) et le fantôme hagiologique», dans E. Воzóку éd., Hagiographie, idéologie et politique au Moyen Âge en Occident, Turnhout, 2012, p. 9-33 (p. 31).

5. R. McMullen, «What Difference did Christianity Make?», Historia, 35 (1986), p. 322343.

6. P. VeYNE «La famille et l'amour sous le Haut-Empire romain», dans ID., La Société romaine, Paris, 2001, p. 108.

7. M. Foucault, Histoire de la sexualité. 3. Le souci de soi, Paris, 1984, p. 177-192. A. CAMERon, «Redrawing the Map: Early Christian Territory after Foucault», Journal of Roman Studies, 76 (1986), p. 266-271 (p. 266) souligne l'importance des Aveux de la chair, quatrième tome annoncé de l'Histoire de la sexualité, dédié aux débuts du christianisme. B. TeuBER, «Chair, ascèse et allégorie sur la généalogie chrétienne du sujet désirant selon Michel Foucault», Vigiliae Christianae, 48 (1994), p. 367-384.

8. A. CAmERon, «Redrawing the Map...»; voir aussi l'introduction de ce volume, n. 13. 
Pour ce faire, elle donne à ce terme la définition suivante: «Il y a sexualité lorsque les actes sexuels et les discours sur l'amour et le désir se rencontrent ${ }^{9}$.» Or, au Moyen Âge, ceci était une question centrale et, si les catégories d' «homosexuels » ou d' «hétérosexuels » n'existaient pas ${ }^{10}$, le fait d'avoir ou non des relations sexuelles était sans doute un aspect déterminant pour l'identité des personnes ${ }^{11}$ : c'est le cas pour les moines, qui se définissent par leur continence, mais aussi pour les couples. L'identité de l'individu est étendue, sinon déterminée, par les relations sexuelles qu'il peut entretenir et, théoriquement, via la constitution d'un couple qui ne fait plus qu'une chair: c'est le fameux principe de l'una caro, quand les conjoints constituent la même chair ${ }^{12}$. Laurent Barry, qui reprend la démonstration de Paul Veyne dans le début de sa réflexion sur l'una caro $^{13}$, la rapproche de 1 ' «invention du couple ${ }^{14} »$. Il souligne cependant l'importance de l'effort doctrinal chrétien qui adapta son discours de façon à concilier les exigences évangéliques et les structures de parenté de la Rome tardo-antique ${ }^{15}$, et qui influença par la suite, sinon les structures de la parenté, du moins les relations à l'intérieur du couple. Il y a bien en effet des mutations essentielles qui se produisent sous l'influence de ces efforts doctrinaux et des idéaux contradictoires véhiculés alors par le discours chrétien sur le mariage ${ }^{16}$, même si ces mutations ne sont pas l'origine de la valorisation du couple conjugal ${ }^{17}$.

9. R. M. Karras, «Sexuality in the Middle Ages», dans P. Linehan, J. L. Nelson éd., The Medieval World, Londres, 2003, p. 292; voir plus largement EAD., Sexuality in Medieval Europe. Doing unto others, New York, 2005.

10. R. Stone qualifie uniquement des actes et non des personnes d'homo- ou hétérosexuels pour l'époque carolingienne: Morality and Masculinity in the Carolingian Empire, Cambridge, 2012, p. 281. Sur la fin de l'Antiquité: E. CANTARELLA, Secondo natura. La bisessualità nel mondo antico, Milan, 1999, p. 239-266.

11. R. M. KarRas, «Prostitution and the Question of Sexual Identity in Medieval Europe», Journal of Women's Studies, 11 (1999), p. 159-177.

12. F. HérItiER, Les Deux Scurs et leur mère. Anthropologie de l'inceste, Paris, 2012, p. 83-85.

13. L. BARry, La Parenté, Paris, 2008, p. 488-493, 523-526.

14. Ibid., p. 490, 525; E. Porqueres I Gené, «Cognatisme et voie du sang. La créativité du mariage canonique», L'Homme, 154/155 (2000), p. 335-356 (p. 342-343); voir aussi J. GAUDEMET, Le Mariage en Occident, Paris, 1987, p. 212 à propos de l'affinitas ex copula illicita .

15. L. BARrY, La Parenté..., p. 505-506.

16. K. CoOper, The Virgin and the Bride. Idealized Womenhood in Late Antiquity, Cambridge, 1996.

17. La difficulté, pour les Pères, est de concilier la nécessité du couple et de la procréation exprimée dans la Bible avec la valorisation de la chasteté (Tertullien tente de contourner le paradoxe dans son traité $\grave{A}$ sa femme). Les efforts des Pères pour construire le couple et définir le mariage chrétien sont loin d'être unanimes, et la question de la chasteté n'est pas traitée de la même manière selon la nature des couples: il y a davantage une multiplicité de cas qu'une théorie abstraite. Voir, au-delà de la multitude d'études sur les Pères, la variété des interprétations de Paul exposée dans 
L'aspiration à la virginité ascétique qui connaît un essor à la fin de l'Antiquité ${ }^{18}$ a pu correspondre à une volonté de transgresser son genre, voire d'atteindre un troisième genre, non seulement par le refus de la sexualité, mais aussi par des pratiques qui ont pour but d'effacer les signes ou les attributs de genre ou de sexe (femmes qui se taillent les cheveux et refusent les vêtements féminins, castration...), un phénomène bien étudié par Matthew Kuefler ${ }^{19}$. Ces choix ne correspondent pas exactement à ceux des couples chastes (ou plutôt de leurs hagiographes): chacun conserve bien son identité d'homme ou de femme, les femmes se décrivant, dans plusieurs de nos récits, comme épouses du Christ $^{20}$. Dans les récits du haut Moyen Âge, aucun accent particulier n'est mis sur la sexualité en tant que tentation à surmonter, et les rôles de chacun en tant qu'homme ou femme sont réaffirmés dans le couple (ou dans leur relation avec le Christ, présenté comme le véritable époux de certaines épouses chastes ${ }^{21}$ ): seuls les accomplissements de l'homme en tant qu'ecclésiastique sont d'ailleurs décrits dans la plupart des cas, une fois le moment du mariage passé. Bien qu'il soit possible que certains des hommes et des femmes décrits dans l'hagiographie du haut Moyen Âge aient eu les mêmes aspirations à dépasser leurs désirs, les narrateurs choisissent de laisser cet aspect de côté. De ce point de vue, les couples chastes représentés dans la Gaule du haut Moyen Âge se distinguent des couples dénoncés par les Pères, qui voyaient cohabiter des hommes (souvent des clercs) et des femmes (dites virgines subintroductae, et en grec syneisaktoi ou agapetae) qui disaient s'entraider mais aussi affirmer leur supériorité par leur lutte perpétuelle face à la tentation de la chair (essentiellement aux III $^{\mathrm{e}}$ et IV siècles).

l'antibiographie par J. Albert Harrill, Paul the Apostle. His Life and Legacy in their Roman Context, Cambridge, 2012, part. p. 106-115.

18. C. Angelidi, «Virginité ascétique: choix, contraintes et imaginaire ( $\mathrm{IV}^{\mathrm{e}}-\mathrm{VII}^{\mathrm{e}} \mathrm{s}$.)», Comportamenti e immaginario della sessualità nell'alto medioevo, Settimane di Studio del centro italiano di studi sull'alto medioevo, Spolète, 2006, p. 675-695.

19. Je remercie Matthew Kuefler des réflexions échangées lors de sa participation à la session «Cross Dressing and Gender Transgression in the Middle Ages» organisée par Irene Barbiera lors du $6^{\text {e }}$ congrès de la Società Italiana delle Storiche (Padoue, 14 février 2013). Voir: M. KuefLer, The Manly Eunuch. Masculinity, Gender Ambiguity and Christian Ideology in the Late Antiquity, Chicago, 2001 ; G. Clark, Body and Gender, Soul and Reason in Late Antiquity, Farnham, 2011 ; J. ReISDOERFER, «C'est l'habit qui fait le moine. Édition de la version valenciennoise de la Vita Sanctae Euphrosynae (BHL 2722)», Zeitschrift für antikes Christentum, 15/2 (1997), p. 227-248 (p. 227 pour une large bibliographie). On trouve une seule femme vêtue en homme et entrée au monastère dans la Gloire des Confesseurs (16), Papula.

20. Ainsi dans l'histoire des Deux Amants: GréGoire de Tours, Dix Livres d'Histoire, I, 47 (éd. B. Krusch, W. Levison, M.G.H., SSRM 1, 1², Hanovre, 1951, p. 31) et Gloire des Confesseurs, 31 (éd. B. Krusch, M.G.H., SSRM 1, 2, Hanovre, 1885, p. 767).

21. Expression qui n'est usitée que pour les vierges consacrées habituellement, et qui est utilisée par la femme d'Injuriosus qui lui offre de partager la dot de son époux céleste: «ego partem tribuam dotis, quam promissam habeo ab sponso domino meo Iesu Christo » (Dix Livres d'Histoire, ibid.). 
Plusieurs textes conciliaires et patristiques de cette époque dénoncent en effet l'habitude prise par certaines femmes ayant dédié leur virginité au Christ de vivre avec un homme ayant également fait le vœu de demeurer chaste. Ces unions, dont les protagonistes affirment se comporter comme frère et sœur, leur semblent dangereuses non seulement parce qu'elles suscitent des racontars, mais parce que nombre de ces couples ne seraient pas capables de respecter leur parole. Jérôme souligne ainsi que certains «ayant fait semblant, d'une façon tout à fait sérieuse au début, d'habiter avec des sœurs, ont été pris en faute à la longue, la sœur étant devenue enceinte des œuvres du frère ${ }^{22}{ }$. Il semble que ce phénomène des virgines subintroductae ait été assez répandu ${ }^{23}$, même s'il faut sans doute renoncer à en chercher des traces dans les épîtres de saint Paul comme le fit Hans Achelis à propos de I Cor. $7^{24}$. Les études sur le sujet se sont multipliées au début du $\mathrm{Xx}^{\mathrm{e}}$ siècle, visant à établir un corpus le plus large possible ${ }^{25}$. Pierre de Labriolle se demanda même si un certain nombre de décisions conciliaires des premiers siècles du christianisme ne concernait pas le mariage spirituel plutôt que le concubinage des clercs ${ }^{26}$. Cette pratique, comme les attaques des Pères, fut davantage répandue en Orient qu'en Occident ${ }^{27}$ : Jean Chrysostome est sans doute, ainsi, celui dont les écrits furent les plus virulents (notamment dans deux traités rédigés dans les années 380 ou 390 ${ }^{28}$. Les assertions des subintroductae, qui prétendent prouver leur valeur en résistant face au désir charnel, sont repoussées par le Père. Jean Chrysostome souligne d'ailleurs que l'absence de passage à l'acte perpétue la présence du désir, alors que les époux qui ont consommé leur union se lassent l'un de l'autre avec le temps ${ }^{29}$. En Occident,

22. JÉrÔMe, Lettre 22, 13-14 (JÉrôME, Lettres, éd. et trad. J. Labourt, Paris, 1949-1963, vol. 1, p. 143).

23. H. A. Sebold, «Spiritual Marriage in the Early Church: a Suggested Interpretation of I Cor. 7, 36-38», Concordia Theological Monthly, 30 (1959), p. 176-184 (p. 184).

24. H. Achels, Virgines subintroductae. Ein Beitrag zur I. Cor. 7, Leipzig, 1902.

25. A. JÜLICHER, «Die geistlichen Ehen in der alten Kirche», Archiv für Religionsgeschichte, 7 (1904), p. 373-386.

26. P. DE LABRIOLLE, «Le “mariage spirituel” dans l'Antiquité chrétienne», Revue historique, 137 (1921), p. 204-225.

27. Il faut bien entendu demeurer conscients de la diversité des vues des Pères sur la question de la femme, de la virginité, de la chasteté, notamment entre Orient et Occident: Joyce E. Salisbury (Church Fathers. Independent Virgins, Londres, 1991, p. 11-55) marque surtout la spécificité d'Augustin. Pour une vue plus complète, se référer à P. BRown, Le Renoncement à la chair, Paris, 1995.

28. E. A. Clark, «John Chrysostom and the Subintroductae», Church History, 46/2 (juin 1977), p. 171-185.

29. Jean Chrysostome, Adversus eos qui apud se habent subintroductas virgines, 1 et 3 (JEAN Chrysostome, Les Cohabitations suspectes. Comment observer la virginité, éd. et trad. J. Dumortier, Paris, 1955). 
c'est Jérôme qui s'attaque en particulier aux couples chastes, ou plutôt en réalité aux femmes de ces couples, qu'il qualifie de meretrices univirae ${ }^{30}$. Il valorise en revanche les liens d'amitié exclusifs qui peuvent exister entre deux abstinents, tant qu'ils ne cohabitent pas, tels lui-même et la veuve Paula ${ }^{31}$. La pratique de la cohabitation comme épreuve à surmonter par l'ascète se retrouve explicitement au haut Moyen Âge dans la description des pratiques $\left\langle\right.$ celtiques $^{32} »$. Le choix du mariage chaste entretient un certain rapport avec l'esprit des martyrs dans ce contexte ${ }^{33}$ : il s'agit de prouver sa foi en s'exposant à une épreuve, celle du désir, un peu comme les saintes martyres résistaient aux épreuves comportant une menace sexuelle (tel le lupanar ${ }^{34}$ pour Agnès ${ }^{35}$ ).

Les succès conjoints de l'ascétisme et de la morale conjugale, au $\mathrm{III}^{\mathrm{e}}$ et surtout au $\mathrm{IV}^{\mathrm{e}}$ siècle, expliquent sans doute l'attrait pour un type d'union qui démontre l'aspect paradoxal de ces aspirations. Cependant, les textes hagiographiques de la toute fin de l'Antiquité et du haut Moyen Âge qui traitent du mariage chaste ne mettent guère en valeur cet aspect. En effet, le mariage est alors présenté comme une obligation sociale qui est acceptée, et la mise en scène de la réunion des deux époux dans le lit conjugal n'est plus conçue prioritairement comme une épreuve physique, mais comme un moment de dialogue décisif.

\section{Pressions familiales et volonté du couple au vi ${ }^{\mathrm{e}}$ siècle}

Le discours hagiographique présente évidemment ces couples comme exceptionnels, comme des modèles de vertu, mais ils demeurent tout à fait en marge des exemples de vie destinés aux couples laïques. Alors même que le nombre de Vies évoquant une union chaste est limité, celles-ci présentent bien un idéal révélateur: même si le couple mène une vie séparée, ou si sa vie commune

30. JÉRÔME, Lettre 22, 14.

31. M. KUEFLER, The Manly Eunuch..., p. 200-203.

32. L. Gougaud, «Mulierum Consortia: étude sur le syneisaktisme chez les ascètes celtiques», Ériu, 9 (1921/1923), p. 147-156; R. ReYNolds, «Virgines subintroductae in Celtic Christianity», Harvard Theological Review, 61 (1968), p. 547-566.

33. Problèmes sur la datation et le public des Passions, en particulier de sainte Agnès: F.-X. Romanacce, «Construction et fonction du récit dans la littérature martyriale. L'exemple de la Passio Perpetuae», dans M. COUMERT et al. éd., Rerum Gestarum Scriptor. Histoire et historiographie au Moyen Âge. Mélanges M. Sot, Paris, 2012, p. 249-259 (p. 250-251).

34. K. Gravdal, Ravishing Maidens. Writing Rape in Medieval French Literature and Law, University of Pennsylvania Press, 1991, p. 22-23.

35. Le texte hagiographique le plus copié et qui circule le plus à l'époque mérovingienne comme l'a rappelé M. Heinzelmann, «L'hagiographie mérovingienne. Panorama des documents potentiels», dans M. GoulLET et al. éd., L'Hagiographie mérovingienne à travers ses réécritures, Ostfildern, 2010, p. 27-82 (p. 78-82); C. LANÉRY, «Hagiographie d'Italie, 300-550. I. Les passions latines composées en Italie», dans G. PhilipPart éd., Hagiographies V, Turnhout, 2010, p. 192-203. 
n'est évoquée qu'en passant, des moments clés de la Vie expriment ce que serait un couple parfait, une véritable association et un véritable accord de deux êtres qui unissent leurs volontés et trouvent une parfaite harmonie. C'est dans la nuit de noces et dans la mort que ces couples sont décrits de la façon la plus frappante. Ce type de récit ne se retrouve plus sous les mêmes traits après le très haut Moyen Âge, peut-être à cause de l'importance moindre donnée au consentement dans le mariage. Si la résistance face à la tentation peut être évoquée, ce n'est que de façon marginale. Le thème de la résistance est bien sûr présent, mais plus dans le couple: la prostituée ou le démon déguisé sont des tentateurs (ou plutôt des tentatrices) beaucoup moins ambigus ${ }^{36}$.

Les histoires de couples chastes tirées des œuvres de Grégoire de Tours et des Vies d'Amâtre d'Auxerre mettent en scène, non pas tant un modèle de couples dont les membres auraient transgressé leur genre, que des histoires qui réussissent à concilier l'idéal de dépassement et de virginité et celui de la vie commune. Ces quelques récits composent un équilibre bien difficile, et on comprend qu'il n'y en ait pas eu davantage, et même que Grégoire de Tours les ait contés avec certaines réserves. Ces couples semblent cependant respecter le modèle de masculinité et l'importance du pater familias adoptés par l'idéologie chrétienne, et renvoient aussi aux aspirations à l'ascétisme de la fin de l'Antiquité, même lorsqu'un rôle actif est donné à la femme. Le récit des Deux Amants par Grégoire de Tours voit la femme proposer à son époux de partager la dot céleste de son «époux le seigneur Jésus-Christ», et le mari tenant l'étendard de la croix. Chacun semble donc bien correspondre aux topoi de son genre.

Grégoire de Tours traite à plusieurs reprises du problème de la sexualité et du mariage pour les saints, et en particulier pour les saints évêques. Dans les Dix Livres d'Histoire ${ }^{37}$, le conte des Deux Amants (I, 47), ajouté dans un second temps (et présent dans une version plus réduite dans la Gloire des Confesseurs, 31), précède la fin d'une époque pour Grégoire (la mort de saint Martin) et suit l'histoire des évêques d'Auvergne (en particulier celle d'Urbicus cédant à sa

36. L'exemple de Benoît, conté dans les Dialogues de Grégoire le Grand, est bien connu. Mais le thème de la tentation et de l'ardeur de la jeunesse (Dialogues II, 1 : «in eius pectore amoris flamma») est strictement séparé du saint couple qui passe la nuit sous le même toit (Benoît et sa sœur ne partagent certes pas le même lit, mais ils sont réunis dans la même tombe: Dialogues II, 33-34: «... quorum mens una semper in Deo fuerat, eorum quoque corpora nec sepultura separararet»): Grégoire le Grand, Dialogues II, éd. A. De Vogüé, Paris, 1979, p. 136-141, 232-235. Grégoire le Grand met tout à fait de côté l'existence de la femme de Paulin de Nole, Thérèse (Dialogues III, 1), contrairement à GRÉGOIRE DE Tours qui la dit castissima après l'élévation de Paulin à la charge épiscopale (De la Gloire des Confesseurs, 110).

37. Grégoire de Tours, Libri historiarum X, éd. B. Krusch, W. Levison, M.G.H., SSRM I. 1, Hanovre, 1951, p. 30. 
femme qui l'a supplié de passer une nuit avec lui [I, 44], puis celle de Népotien qui a brisé ses fiançailles [I, 46]).

Dans son œuvre hagiographique, la Gloire des martyrs ${ }^{38}$ évite totalement le sujet, non seulement des évêques face au mariage, ce qui est bien normal, mais même de la tentation sexuelle. La seule épouse d'évêque qui y apparaisse est celle de Sidoine Apollinaire, Placidine, mais sans que son statut et son style de vie soient en rien discutés $(I, 65)$. Des épouses laïques apparaissent en revanche en tant que confidentes ou bonnes conseillères de leur époux (I, 48; I, 80; II, 14). La Gloire des Confesseurs ${ }^{39}$, en plus de l'histoire des Deux Amants, nous rapporte le plus grand nombre de cas, avec la plus grande variété, et selon une gradation chronologique qui se veut sans doute, aussi, une gradation vers un aspect de plus en plus patent des paradoxes qui existent entre mariage et cohabitation pour l'évêque et son épouse ${ }^{40}$. Tout d'abord l'évêque d'Autun Rétice se marie tout en demeurant chaste en secret, et il ne devient évêque qu'une fois devenu veuf (75). Son successeur Simplicius mène de la même façon une vie chaste auprès de son épouse, qui veut continuer à vivre et même à dormir auprès de lui une fois qu'il a été élu évêque. Celle-ci doit accomplir un véritable miracle en mettant le feu à ses vêtements sans se consumer (comme elle n'est pas consumée par le désir). Mais elle y parvient, et mène plus de mille hommes à la conversion (76). L'évêque de Nantes, évoqué quelques chapitres plus loin, est davantage représentatif de ce qui se produit à l'époque de Grégoire : il a apparemment vécu une vie conjugale consommée avec son épouse, mais se sépare d'elle une fois élu (78). Cela rend cette dernière suspicieuse. Le miracle qui survient (un agneau posé sur la poitrine de son époux qui dort) vise à prouver la chasteté de l'évêque alors même qu'il s'est séparé de son épouse. Ce couple n'est donc pas fondé par une décision commune et un accord sans faille comme dans le cas des couples chastes. Au contraire, une fois que le mariage a été consommé, le désir et/ou la suspicion s'installent. Ces récits aux accents merveilleux soulignent en réalité combien, pour Grégoire, ces hommes et ces femmes qui tentent l'aventure du mariage spirituel appartiennent à un passé révolu. Ce genre de miracle n'apparaît plus dans son œuvre lorsqu'il présente les épouses des évêques de son époque ${ }^{41}$.

Dans la Vie des Pères ${ }^{42}$, on n'a plus affaire à des couples chastes, mais à de saints hommes qui fuient le mariage (Gall 6,2; Patrocle 9, 1; Venant 16,

38. ID., Liber de gloria martyrum, éd. B. KRUSCH, M.G.H., SSRM, I. 2, Hanovre, 1885, p. 484561.

39. ID., Liber de gloria confessorum, M.G.H., SSRM, I. 2, p. 744-820.

40. S. Joye, «Grégoire de Tours et les femmes. Jugements portés sur les couples laïcs et ecclésiastiques», dans C. LA RoccA éd., Agire da donna, Turnhout, 2007, p. 83-84.

41. S. Joye, «Grégoire de Tours et les femmes...», p. 86.

42. GRÉGoIRE De Tours, Liber de uitae Patrum, M.G.H., SSRM, I. 2, p. 661-744. 
1; Liphard 20,1). Ce sont les relations avec les parents du saint qui prévalent: il n'y a pas de véritable rébellion cependant. Le père de Gall est finalement convaincu qu'il doit devenir ecclésiastique; Patrocle refuse le mariage mais après la mort de son père; et Liphard est rappelé à l'obéissance par ses parents qui le fiancent, mais ceux-ci meurent opportunément avant le mariage. Grégoire de Tours évoque encore un autre type de couple dont il met en valeur la chasteté, ou plutôt l'usage raisonné de la sexualité. C'est l'idée plus commune du bon usage de la sexualité dans le mariage. Ici, le roman familial de Grégoire prend le dessus: il valorise une famille épiscopale, la sienne. La procréation est donc nécessaire à l'existence de cette famille, non pour son héritage terrestre, mais pour que naissent de grands évêques. Grégoire, évêque de Langres, et Armentaria mènent ainsi une vie chaste, sauf pour produire une descendance: ils n'ont que des fils et Grégoire ne devient évêque qu' après la mort de son épouse (8). Artémie, la femme de Florentius, refuse que son mari devienne évêque de Genève car elle est enceinte: elle affirme à son mari qu'elle a bénéficié d'une révélation au sujet de son fils, qui deviendrait un très grand évêque, en l'occurrence Nizier de Lyon (7). Grégoire de Tours insiste bien sur l'affection et la modération de ces couples: Grégoire de Langres n'a jamais brûlé pour une autre femme que son épouse, et Nizier et sa mère Artémie forment en quelque sorte le couple parfait, Nizier demeurant avec sa mère après la mort de son père et son entrée dans le clergé. Ainsi l'écrasante demande des parents nobles pour que soit prolongée leur lignée est sanctifiée ici par Grégoire, car elle concerne non un héritage terrestre mais un héritage céleste ${ }^{43}$.

Si le Père de Gaiffier a inclus dans sa liste de couples chastes ceux qui le sont devenus après un mariage consommé, les individus dont nous parlerons plus précisément ici s'engagent dans le choix de la chasteté, et même de la virginité, au moment de la formation du couple, soit du mariage ${ }^{44}$. La consommation du mariage est remplacée par une abstinence discutée : la nuit de noces est présentée comme le moment d'un véritable échange et d'une prise de décision commune, d'un consentement, qui fonde réellement le couple mais préside aussi parfois à sa séparation dans les faits.

Ainsi, les femmes semblent pouvoir également exprimer leur volonté face au mariage et à la sexualité dans ces histoires de couples chastes, alors qu'elles ne le font pratiquement jamais dans les autres récits, ni sans doute dans

43. Au IV s., Mélanie demande à son époux Pinien qu'ils demeurent vierges dans le mariage, mais celui-ci réclame un héritier avant de devenir chaste (BHL 5885).

44. B. DE GAIfFIER, «Intactam sponsam reliquens. À propos de la Vie de S. Alexis », Analecta Bollandiana, 65 (1947), p. 157-195 (p. 164-184). 
la réalité. Comme l'a bien rappelé Kate Cooper ${ }^{45}$, une partie du grand succès du christianisme auprès des dames romaines $a u I^{e}$ siècle vient du succès de l'ascétisme féminin, celui-ci allant de pair avec la possibilité d'exprimer une autorité féminine, alors que toute autorité devait être masculine dans leur vie sociale et qu'elles y avaient de moins en moins de place. C'est à cette époque que le thème de l'influence morale de la femme sur son époux se serait développé ${ }^{46}$. De fait, on trouve quelques figures de femmes qui sont à l'origine du refus de la consommation du mariage, même si les Vies de saints laissent souvent une part importante à l'époux dans ce choix (qui revient - presque - toujours au mari lorsqu'il s'agit de cesser la sexualité ou la vie commune au moment d'une entrée dans la vie religieuse) ${ }^{47}$.

L'accord des époux est fondamental. En cela, les couples chastes de l'hagiographie sont de véritables couples, qui fondent leur vie à venir dans une décision commune et, dans une partie des cas, qui entretiennent des relations que l'on pourrait qualifier d'amitié. Augustin rappelait d'ailleurs que priment dans le couple l'accord de la volonté, de la raison et de la sexualité, mais aussi et avant tout l'amitié ${ }^{48}$. S'il semble être à l'origine de l'idée de devoir conjugal et de l'importance de l'usus tori ${ }^{49}$, il estime aussi que la charité est mieux partagée chez les époux qui décident d'un commun accord de devenir continents tout en continuant leur vie commune, « libres de profiter d'une intimité sincère ${ }^{50}$. Ainsi, à la fin de l'Antiquité, la sexualité représentait, selon les mots de Peter Brown, une «facette des relations sociales humaines», un devoir social vis-à-vis de sa famille et de la cité, et un "problème pour la volonté » ${ }^{51}$. L'accomplissement du devoir conjugal apparaissait, à travers de nombreux écrits chrétiens, comme une nécessité qui permettait non seulement de préserver le mari des tentations extérieures, mais aussi éventuellement de l'influencer afin qu'il mène une vie

45. K. COOPER, «Poteri invisibili: la matrona cristiana tra obsequium e autorità legittima all'epoca di Sant'Augusto», dans C. LA Rocca éd., Agire da donna..., p. 12; voir aussi: F. E. Consolino, «Modelli di comportamento e modi di sanctificazione per l'aristocrazia femminile d'Occidente», dans A. Giardina éd., Società romana e impero tardoantico I. Istituzioni, ceti e economia, Bari, 1986, p. 273-306; E. A. CLARK, «Ascetic Renunciation and Feminine Advancement: a Paradox of Late Ancient Christianity », Anglican Theological Review, 6 (1981), p. 240-257.

46. K. COOPER, «Insinuations of Womanly Influence: an Aspect of the Christianization of the Roman Aristocraty », Journal of Roman Studies, 82 (1992), p. 150-164.

47. Ce n'est pas le cas dans la Vie de Cécile (BHL 1495) ou le récit des Deux Amants par Grégoire de Tours.

48. P. Brown, Le Renoncement à la chair..., p. 481-485.

49. J. A. Brundage, Law, Sex and Christian Society in the Medieval Europe, Chicago, 1987, p. 93.

50. P. Brown, Le Renoncement à la chair..., p. 483. Voir Augustin, Lettre 31, 6, à propos de Paulin de Nole et de sa femme Thérèse.

51. P. Brown, Le Renoncement à la chair..., p. 465. 
plus conforme aux préceptes chrétiens. Durant le haut Moyen Âge en Occident, les moralistes qui s'adressent aux reines chrétiennes mariées à des païens ou à des hérétiques préconisent d'ailleurs qu'elles usent justement de la chambre à coucher pour convaincre leur époux de se convertir au christianisme ${ }^{52}$.

C'est jusque dans le lit conjugal que l'hagiographe met en scène le couple saint lorsqu'il choisit d'évoquer son refus de consommer le mariage ${ }^{53}$. Nous ne pouvons développer ici les discussions qui eurent lieu, à la fin de l'Antiquité et au très haut Moyen Âge, sur la place de la consommation dans le mariage ou sur le mariage de Marie et Joseph ${ }^{54}$. Cette question n'entre guère dans les préoccupations de nos hagiographes, qui ne se privent cependant pas de souligner la réalité du couple, véritable consortium dans la mort comme dans la vie ${ }^{55}$. Le discours sur le refus du désir et des séductions mondaines évoque en fait d'autres plaisirs, certes spirituels, mais qui impliquent aussi les sens, la beauté, ainsi qu'un consentement et un désir communs, discutés par les époux qui se destinent à devenir un couple dans l'esprit en renonçant à la cohabitation ou en gardant leur chasteté secrète face au monde et, surtout, aux pressions familiales. Le mariage chaste est presque toujours présenté en effet comme une réponse aux pressions de la famille qui souhaite que son unique héritier se marie et lui donne une descendance pour que perdure l'héritage familial. Les discussions sur l'obéissance et le choix sont donc essentielles pour ces individus qui se soumettent à la fois à la lettre de l'ordre donné par leurs parents, et à l'esprit de la loi divine et de leurs propres aspirations.

L'exemple d'Amâtre (Amator, Amour), évêque d'Auxerre mort en 418, met bien en valeur ces derniers éléments. Tout ce qui concerne le mariage, la nuit de noces et les aspects miraculeux accompagnant l'accord des époux est largement développé, bien que quelques éléments nous éloignent déjà du couple qui partage une vie entière de chasteté: l'officiant remplace secrètement, et de son plein gré, la bénédiction du mariage par une bénédiction diaconale, et les époux ne mènent pas une vie commune après la nuit passée ensemble dans le lit

52. J. L. Nelson, «Queens as Converters of Kings in the Early Middle Ages», dans C. LA Rocca éd., Agire da donna..., p. 95-107.

53. En particulier dans la Vie d'Amâtre, $c f$. infra.

54. Marie est très présente dans l'homilétique, en particulier chez Ambroise ou Augustin, et de nombreuses études lui ont déjà été consacrées. Voir entre autres D. LhUILLIER-MARTINETTI, L'Individu dans la famille à Rome au Ive siècle d'après l'œuvre d'Ambroise de Milan, Rennes, 2008, p. 40-45; P. L. ReYnolds, Marriage in the Western Church, Boston, 2001, p. 338-344.

55. Rétice d'Autun par GréGoIre de Tours, Gloire des Confesseurs, 75 (éd. B. Krusch, M.G.H., SSRM 1, 2, Hanovre, 1885, p. 342): « ut quos unius castitatis dilectio uno conservavit in toro, unius retineat sepulcri consortium»; Vie d'Amâtre par ÉTIENNE D'AFRIQUE : «Thorus igitur coniugalis, strati tantum communis consortium existat, non in mundanis illecebris » (Vita Amatoris, BHL 356, AA SS Mai I, Anvers, 1680, p. 52-53). 
des noces. La Vie d'Amâtre a été mise par écrit à la fin du VI siècle par Étienne d'Afrique, à la demande de l'évêque Aunarius ${ }^{56}$, et dans les Gestes des évêques d'Auxerre, dont la notice date des années 870 (le couple est évoqué dans la notice d'Amâtre et dans celles de ses prédécesseurs Valérien et Élade ${ }^{57}$ ).

Dans la version datée des alentours des années 600, le récit est bien entendu plus développé. On remarque une attention particulière à l'opposition aux désirs des parents et à la volonté de ceux-ci de marier les jeunes époux pour avoir une descendance, d'autant qu'Amâtre serait fils unique ${ }^{58}$. Les éléments qui marquent la richesse et le détail de la cérémonie sont nombreux. On sait que la jeune fille semble déjà décidée, elle aussi, à choisir la voie de la virginité. Arrive le jour où ils doivent partager la même habitation. Les noces sont célébrées selon les «rites des Romains». Valérien est invité à participer aux noces et à les confirmer par une prière, mais il donne la bénédiction des diacres et non celle des époux. Personne ne le comprend cependant dans l'assemblée, et c'est seulement dans le lit nuptial («alors que le soir était arrivé et que le temps de l'union était venu $\left.{ }^{59} »\right)$ qu'Amâtre parle avec celle qui est son associata virgo. Et son associatus juvenis ajoute à sa couronne de pudeur la palme de la chasteté. À tous ces termes, qui soulignent une réelle union des deux jeunes gens même s'ils ont reçu une bénédiction diaconale, s'ajoute celui du consortium, qui s'accomplit dans le lit conjugal, mais sans concerner les séductions mondaines ${ }^{60}$. Une douce odeur de sainteté se répand déjà.

Un ange apparaît alors qu'ils se sont endormis: Amâtre sait que c'est la réalité alors que sa jeune épouse pense qu'il s'agit d'un rêve (on est dans le schéma inverse de celui de la Vie de Cécile, qui convainc son mari Valérien de ne pas l'épouser car elle voit un ange prêt à la tuer s'il la touche, Valérien ne voyant l'ange qu'une fois que Cécile lui a signalé sa présence) ${ }^{61}$. Les noces sont bien censées être passées, mais en même temps les jeunes gens sont demeurés immaculati atque incontaminati, et c'est la bénédiction des lévites qui prend

56. Vita Amatoris (BHL 356), AA SS Mai I, Anvers, 1680, part. p. 52-53. I. RéAL, Vies de saints, vie de famille, Turnhout, 2001, p. 170-177.

57. Les Gestes des évêques d'Auxerre. I (éd. et trad. M Sot et al., Paris, 2002, p. 20-29).

58. «Non voluntas mea sed parentum persuasit frequens impulsio»: Vita Amatoris, AA SS Mai I, Anvers, 1680, p. 52.

59. «Cum iam advesperasceret et copulationis tempus urgeret»: Vita Amatoris, AA SS Mai I, Anvers, 1680, p. 53.

60. Vie d'Amâtre par ÉTIENNE D'AfRiQue: «Thorus igitur coniugalis, strati tantum communis consortium existat, non in mundanis illecebris ».

61. BHL 1495 (par Arnobe le Jeune) : C. LANÉRY, «Hagiographie d'Italie, 300-550. I...», p. 80-88. Cette Passion a un aspect très catéchétique et l'auditoire visé devait donc bien être large et laïque autant qu'ecclésiastique. Ce thème est aussi à rapprocher des histoires de Julien et Basilissa ou de Chrysanthe et la vestale Darie (BHL 1787, 2e moitié du ve s.; C. LANÉRY, ibid., p. 145). 
le dessus, même si en réalité un nouveau rituel est nécessaire. Celui-ci sépare cette fois le couple: c'est la tonsure d'Amâtre et la prise de voile de Marthe (qui ne réapparaît plus dans la suite du texte). Amâtre est enterré auprès de ses successeurs au Mont Artre.

L'aspect paradoxal de la réunion, comme dans une même chambre, des deux époux se retrouve cependant pour certains dans la mort qui les rapproche éternellement. Dans les histoires qui valorisent réellement un couple, et pas plus l'homme que la femme, la pureté du couple se traduit par cette cohabitation qui demeure intime tout en restant évidemment chaste. Le miracle qui permet le rapprochement des tombes des époux, comme dans un lit commun, est bien sûr mieux conté dans l'histoire des Deux Amants rapportée par Grégoire de Tours, qui dit que leurs tombeaux se sont rapprochés «sans doute pour que ces deux êtres, que le ciel réunissait, n'eussent pas leurs corps séparés dans le monument où ils étaient ensevelis ${ }^{62} \gg$. Cette histoire, dont le souvenir était lié à un lieu particulier, avait sans doute un impact populaire fort, comme Grégoire le souligne lui-même, et mêlait plus vie terrestre et amour céleste que les récits davantage destinés à un public ecclésiastique. Cependant, ce n'est pas le seul récit de Grégoire sur le même thème: à la mort de l'évêque Rétice, sa femme demande aussi à être placée dans le même tombeau que lui, «afin que soient joints encore par l'union dans un même sépulcre ceux que l'amour avait conservés dans le même lit d'une même chasteté». Une fois Rétice mort, il est porté au sépulcre de sa femme et les os de celle-ci se déplacent pour lui faire place auprès d'elle.

\section{Des unions de plus en plus difficiles à mettre en valeur}

Dans les Vies plus tardives ${ }^{63}$, on met plus volontiers en avant le refus du mariage (ou les fiançailles brisées), rébellion dirigée contre la volonté parentale de marier les jeunes gens, qui se termine cependant par un acte de réconciliation.

62. GréGOIRE De Tours, Dix Livres d'Histoire, I, 47. (trad. R. LATOuChe, vol. 1, Paris, 1963, p. 67). La sépulture commune et les liens familiaux qui perdurent au-delà de la mort sont essentiels même si par ailleurs les Évangiles indiquent que les ressuscités n'ont plus ni femme ni mari (Luc 20, 27-28). Ainsi débute par exemple l'épitaphe de Dynamius et Euchéria: «Ceci est le tombeau commun d'un couple aimant, car même la mort n'éteint pas l'amour qui aime. Eux qui étaient égaux en affection ils sont encore réunis après les funérailles; un même sentiment et un même lieu les réunissent », et plus loin: «un amour si chaste (castus amor) les unissait que l'un et l'autre partageaient un même esprit et un même cœur» (ce chaste amour ne désigne pas un couple vierge ici, puisque leur petitfils est l'auteur de ces vers. Bien entendu, le terme «chaste» renvoie plus généralement à ce qui est pur et convenable: $c f$. n. 3): M.G.H. AA VI/2, p. 194; B. DumÉZIL, «Le patrice Dynamius et son réseau », dans Y. Codou, M. LAuwers éd., Lérins, une île sainte de l'Antiquité au Moyen Âge, Turnhout, 2009, p. 167-193 (p. 192).

63. D. Elliott, Spiritual Marriage: Sexual Abstinence in Medieval Wedlock, Princeton University Press, 1995, p. 63 sq. sur le haut Moyen Âge. 
Comme l'a bien montré Régine Le Jan, les histoires de saintes qui refusent le mariage pour entrer au monastère servent surtout à magnifier la réconciliation finale par la fondation d'un monastère familial, à une époque où les aristocrates francs se mettent à multiplier ce type de fondation ${ }^{64}$. En revanche, l'histoire des couples chastes (ou plutôt des couples vierges) commence par une soumission partielle ou fictive à la volonté des parents (même dans le récit que fait Grégoire au sujet des Deux Amants, si la première phrase indique simplement qu'Injuriosus demande la main de sa future épouse, on apprend ensuite qu'ils sont tous les deux enfants uniques issus de très grandes familles d'Auvergne, et Injuriosus lui-même évoque la volonté de leurs parents). Dans le cas des mariages chastes du très haut Moyen Âge, les saints ne se rebellent pas directement face aux pressions familiales qui les poussent à se marier. Mais aucun bénéfice qui engagerait toute la famille dans la voie de la sainteté ou l'accès au sacré ne vient terminer l'histoire: les parents disparaissent. L'élément important est, d'une part, la virginité conservée (essentielle dans le très haut Moyen Âge où le discours religieux ne met guère en valeur le mariage) et, d'autre part, la mise en valeur d'une décision prise par un couple, dans un dialogue qui souvent n'a pas besoin de la persuasion ${ }^{65}$.

Les couples qui deviennent chastes après avoir mené une vie conjugale incluant la sexualité, en particulier lorsque le mari devient évêque, répondent à une autre logique, qui n'est pas opposée à la nécessité familiale de perpétuer la famille et de la mettre en valeur, au contraire. Mais dans l'idéal, les époux doivent se séparer, tout en demeurant mariés en théorie ${ }^{66}$. En ce qui concerne l'entrée dans la vie monastique des époux (et non la promotion à l'épiscopat), le public des Vies n'est pas le même (ce sont davantage les moines que pour les autres Vies envisagées jusqu'ici) et on observe une évolution à l'époque carolingienne ${ }^{67}$ :

64. R. LE JAN, «Monastères de femmes, violence et compétition pour le pouvoir en Francie au vII ${ }^{\mathrm{e}}$ siècle», dans EAD., Femmes, pouvoir et société dans le haut Moyen Âge, Paris, 2001, p. 103 ; S. JoYe, «I conflitti familiari per la figlia nubile (v-IX secolo)», Genesis, 9/1 (2010), p. 29-54 (p. 45).

65. Les monastères féminins de la Gaule $\mathrm{du} \mathrm{vl}^{\mathrm{e}} \mathrm{s}$. permettent aussi de forger une identité, celle de l'aristocratie gallo-romaine. Les Vies et les textes qui concernent la Provence montrent chez les femmes une école d'ascétisme inspirée des cercles aristocratiques féminins de l'époque de Jérôme. Même si le statut des vierges consacrées est de plus en plus associé à la clôture alors, il faut garder à l'esprit que les monastères demeuraient aussi des lieux ouverts d'échange et de culture: S. GiOANNI, «"Être véritablement moine": les représentations de l'identité ascétique dans la pastorale lérinienne», dans Lérins, une île sainte..., p. 141-165 (p. 154).

66. C. Mériaux, «La séparation des époux et leur conversion à la vie monastique d'après quelques sources hagiographiques du haut Moyen Âge», dans E. SANTinelli éd., Répudiation, séparation, divorce dans l'Occident médiéval, Valenciennes, 2007, p. 53-58.

67. R. Stone, Morality and Masculinity..., note que les moralistes carolingiens ne mettent pas en valeur la vertu sexuelle des nobles francs et que la fuite du mariage et du sexe n'est pas valorisée (p. 284-287, 308-309). L'aspiration à un «ascétisme sexuel» redevient un indice de vertu au $\mathrm{x}^{\mathrm{e}} \mathrm{s}$. : 
Charles Mériaux a bien montré, par exemple, comment la Vie de Wandrille est remaniée au IX $\mathrm{X}^{\mathrm{e}}$ siècle pour évoquer la chasteté des époux, ce qui n'était pas le cas de la Vita prima $^{68}$. Mais le récit de la vie conjugale, assez long dans la Vita prima (qui rappelle Mt 19,5, à propos de l'una caro), est réduit à sa plus courte expression dans la Vita secunda. Si toute cohabitation est devenue impossible une fois la vie religieuse choisie, le rédacteur est également soucieux de ne pas montrer un mariage qui serait cassé, à une époque où la consommation du mariage devient un élément de sa définition. Ce modèle des époux qui entrent dans la vie religieuse est lui aussi bientôt jugé trop ambigu et ne convient plus à l'Église, du moins pour l'épiscopat, d'autant que les couples ne se séparaient pas toujours, ou qu'ils continuaient à partager des intérêts communs ${ }^{69}$. Cette situation déplaisait déjà au plus haut point à un Grégoire de Tours, qui présentait les couples épiscopaux et les couples chastes en général sous un jour positif uniquement quand ils appartenaient à un passé lointain et quasi hérö̈que ${ }^{70}$. La persistance du couple en tant que tel, qui échange conseil, gestion du patrimoine et toit, voire lit commun, est un idéal qui ne peut être exemplaire (malgré l'exemple des Deux Amants qui dorment dans le même lit, main dans la main, le jour de leur mariage, puis couchent pendant des années dans le même lit tout en restant d'« une admirable chasteté ») $)^{71}$.

Ainsi, le motif du couple chaste, sans disparaître pour autant, devient beaucoup moins fréquent et plus suspect: il n'est souvent qu'un thème ajouté

S. AIRLIE, «The Anxiety of Sanctity : St Gerald of Aurillac and his Maker», Journal of Ecclesiastical History, 43 (1992), p. 372-395 (p. 389-392).

68. Vita Wandregisili secunda (av. 830), c. 3 (éd. P. VAN Den Bossche, AA SS Julii. 5, p. 272); C. MériauX, «La séparation...», p. 64-65 ; J. HowE, «The Hagiography of Saint Wandrille (Fontenelle) (Province of Haute-Normandie)», dans M. Heinzelmann éd., L'Hagiographie du haut Moyen Âge en Gaule du Nord, Stuttgart, 2001, p. 127-192 (p. 163-169).

69. Certains cas fort concrets nous évoquent la difficulté de certains évêques à se séparer de leur épouse et de la vie en commun. Ennode de Pavie, dans sa lettre 7,20, interroge à ce sujet l'évêque Maxime, qui vit dans la chasteté depuis son élévation à l'épiscopat de Milan. La correspondance d'Ennode, alors diacre de Milan, révèle d'ailleurs qu'il faisait tout pour se rendre à Pavie retrouver la religieuse Speciosa qui avait été sa femme ou, du moins, sa fiancée (Lettres 2, 3): ENNODE DE Pavie, Lettres, éd. et trad. S. Gioanni, Paris, 2006. Je remercie Stéphane Gioanni pour ces références et pour ses conseils sur l'ensemble de cet article.

70. S. Joye, «Grégoire de Tours et les femmes...», p. 75-94; F. E. Consolino, «Gregorio di Tours, Venanzio Fortunato e le mogli dei vescovi in Gallia», dans R. BARCELlona, T. SARDELla dir., Munera amicitiae, studi di storia e cultura sulla Tarda Antichità offerti a S. Pricoco, Palerme, 2003, p. 75-93.

71. Eucher, futur évêque de Lyon, se retire à Lérins avec sa femme et ses deux enfants: S. GioAnNI, «"Être véritablement moine"... », p. 141-142. Stéphane Gioanni souligne bien qu'une fois encore la perspective est tout autre au $\mathrm{vl}^{\mathrm{e}}$ siècle lorsque le pape Grégoire le Grand désire mettre de l'ordre dans les communautés insulaires où cohabitent hommes et femmes telle Eumorfia (GRÉGOIRE Le Grand, Lettres I. 48, éd. P. Minard, SC 370, Paris, 1991, p. 234). 
après coup en vue de la sanctification de hauts personnages demeurés stériles, tels Henri II et Cunégonde. Il en va de même de l'affirmation tardive de la virginité de Radegonde au $\mathrm{Xv}^{\mathrm{e}}$ siècle, alors qu'à la fin du $\mathrm{XI}^{\mathrm{e}}$ siècle, Hildebert de Lavardin soutient encore que la sainte se soumettait à l'intimité conjugale «pour être agréable à son mari, et non pour le plaisir des sens » et «afin d'éviter à son mari d'être tenté par Satan ${ }^{72}$. Les remarques qui touchent à la chasteté de tels couples sont souvent assez tardives et se démarquent nettement du couple vierge que nous avons évoqué. Si seulement l'un des deux époux est réputé saint, comme c'est le cas pour Radegonde, le modèle n'est pas celui du vœu de chasteté partagée, mais plutôt celui de la martyre, vierge par excellence, ici martyrisée au moyen du mariage (alors que les histoires de saints qui refusent le mariage sont beaucoup plus nombreuses). Si Fortunat la décrit tentant de son mieux de remplir ses devoirs d'épouse (lors des banquets par exemple), il prend soin de la représenter la nuit sortant dès qu'elle le peut du lit, en prétextant les «nécessités de la nature», pour se livrer à des mortifications sur le sol de la chambre conjugale (et priant pour son époux). Les représentations romanes de la Vie de Radegonde ont d'ailleurs insisté sur la parenté avec la figure de la martyre, en montrant Radegonde face à Clotaire comme la martyre devant son juge, puis en faisant alterner les moments où elle est comme enfermée et ceux où elle doit paraître en public (ce qu'a bien montré Magdalena Carrasco ${ }^{73}$ ).

Le thème du mariage qui n'est pas consommé est célébré dans la suite du Moyen Âge au travers de la figure de saint Alexis - qui, dans la Vie rédigée à la fin du $x^{e}$ siècle (BHL 286), accomplit tous les rituels qui font de sa fiancée son épouse. Cependant, il n'y a plus alors de confusion des types de sainteté ni des modèles de vie. Alexis persuade bien son épouse de ne pas consommer l'union, malgré les admonestations de son père, et le fait que les parents d'Alexis euxmêmes aient choisi de rester chastes après sa naissance. Cependant, celle-ci, comme une bonne épouse, reste «dans la maison de son mari» même s'il quitte immédiatement ce foyer. Si elle demeure aimante et pleure ensuite la mort de son époux, elle n'est pas associée à son aspiration à une vie spirituelle et se contente de demeurer obéissante et fidèle ${ }^{74}$ : le mélange des genres entre vie religieuse

72. Hildebert de Lavardin, Vie de sainte Radegonde, 11; voir en dernier lieu, avec bibliographie: J. DALARUN, «Dieu changea de sexe, pour ainsi dire » : La religion faite femme $\left(\mathrm{XI}^{e}\right.$ $X V^{e}$ s.), Münster, 2008, p. 84-86.

73. M.E. CARRASCO, «Spirituality in Context: The Romanesque Illustrated Life of St Radegund of Poitiers (Poitiers, Bibl. Mun., MS 250)», The Art Bulletin, 72/3 (1990), p. 414-435 (p. 420).

74. H. DriJvers, «Die Legende des heiligen Alexius und der Typus des Gottesmannes im syrischen Christentum», dans M. Sснмidt, C. F. GeYer éd., Typus, Symbol, Allegorie bei den östlichen Vätern und ihren Parallelen im Mittelalter, Regensbourg, 1981, p. 187-217; M. Perugi, La Vie de saint Alexis, Genève, 2000, p. 18-19; B. DE GAIFFIER, «Intactam sponsam reliquens... », part. p. 164166 et 185-195. 
et vie conjugale est alors réduit au minimum. Le partage du lit conjugal ne peut plus être associé à la virginité.

Sylvie Joye - Université de Reims, Département d'Histoire, 57 rue PierreTaittinger, 51096 Reims Cedex

\section{Couples chastes à la fin de l'Antiquité et au haut Moyen Âge}

L'article évoque pour commencer la figure du couple resté vierge après le mariage dans l'hagiographie du très haut Moyen Âge. Il rappelle les paradoxes de la valorisation simultanée de l'ascétisme et de l'amour conjugal à la fin de l'Antiquité. L'article analyse ensuite plus précisément la mise en scène de ces couples vierges, en particulier dans la Vie d'Amâtre et dans les œuvres de Grégoire de Tours. Le récit du déroulement de la nuit des noces, des preuves de la virginité des époux, de la mise au tombeau permettent d'entrevoir à la fois l'idéal du mariage et celui de l'ascétisme, qui deviennent de plus en plus inconciliables. À partir de l'époque carolingienne et même dès le vII ${ }^{\mathrm{e}}$ siècle, ce modèle disparaît quasiment, ou ne subsiste que sous une forme bien aseptisée, qui gomme toute l'intimité de la cohabitation.

chasteté - virginité - hagiographie - ascétisme - sexualité

\section{Chaste Couples in Late Antiquity and in the Early Middle Ages}

This paper first evokes the married couples which are reputed to live a virginal union in the hagiography of Early Middle Ages. It stresses anew the paradoxes of the simultaneous valuation of asceticism and conjugal love during Late Antiquity. This paper analyzes then more acutely the depiction of these virgin couples, in particular in Amator's first Life and in the writings of Gregory of Tours. The narrative of the chaste wedding night, the proofs of the spouses' virginity or the shared tomb reveal simultaneously the ideals of marriage and asceticism, which become more and more irreconcilable. From Carolingian times and even from the seventh century onward, these virgin couples almost disappear in the Saints' Lifes, which put aside all the intimacy of a cohabitation. chastity - virginity - hagiography - asceticism - sexuality 
